\title{
Les antibiotiques inhibiteurs de la synthèse protéique
}

L'analyse du mode d'action des antibiotiques inhibiteurs de la synthèse protéique a permis de préciser le fonctionnement du ribosome dans la réalisation des différentes étapes : initiation de la traduction, élongation de la chaîne protéique et terminaison. De nombreuses résistances aux antibiotiques sont dues à des mutations des gènes chromosomiques, notamment ceux codant pour les ARN ribosomiques $16 S$ et $23 S$. Cela renforce l'idée que les ARN pourraient participer en tant que tels à la catalyse de diverses réactions du processus de traduction.

\section{Carlo Cocito Mario Di Giambattista}

\section{ADRESSE}

C. Cocito: professeur ordinaire à l'universile de Louvain. M. Di Giambattista : chercheur du Fonds national de la recherche scientifique. Unité de microbiologie générale et de génétique moléculaire, faculté de médecine, ICPUCL 7449, avenue Hippocate 75, B1200 es antibiotiques ont connu un grand essor vers la moitié de notre siècle, suite à leur emploi dans la thérapie des maladies infectieuses. D'autre part, l'utilisation des antibiotiques pour élucider des fonctions cellulaires et les bases de la résistance bactérienne, a grandement contribué aux progrès de la biologie moléculaire. Certains antibiotiques sont en fait devenus des réactifs précieux pour suivre des activités enzymatiques et disséquer certaines voies métaboliques [1-3]. Les antibiotiques les plus étudiés sont ceux qui interfèrent avec les réactions d'édification des macro-molécules (ADN, ARN, protéines) ou l'assemblage des membranes et des parois cellulaires. Notre article se portera sur les études récentes, visant à éclaircir le mécanisme d'action des principaux inhibiteurs de la synthèse des protéines et les relations entre la structure et les fonctions de leur cible, le ribosome (Tableau I). Les différentes approches expérimentales peuvent se résumer comme suit: (a) les systèmes acellulaires de synthèse protéique ; (b) le marquage des sites de fixation à l'aide d'antibiotiques modifiés; (c) l'analyse des modifications ribosomales qui sont responsables de la résistance; et (d) l'effet protecteur, exercé par certains antibiotiques, sur les séquences de l'ARNr exposées à des réactifs chimiques. Signalons qu'un article publié dans cette même revue [4] décrit en détail l'enchaînement des acides aminés en polypeptides sur la matrice d'ARNm, la molécule vectrice de l'information génétique. Une série de facteurs de nature protéique, catalysant l'initiation (IF), l'élongation (EF) et la terminaison (RF), ainsi que les aminoacyl-ARNt et les ribosomes $70 \mathrm{~S}$ participent à cette biosynthèse. Ces derniers sont constitués de deux sous-unités, $30 \mathrm{~S}$ et $50 \mathrm{~S}$, se dissociant lors de la terminaison et se réassociant lors de l'initiation [5].

\section{Antibiotiques inhibiteurs de la petite sous-unité ribosomique}

La sous-unité $30 \mathrm{~S}$, impliquée dans le démarrage des chaînes polypeptidiques [6], participe également, en union avec la sous-unité $50 \mathrm{~S}$, à l'élongation. Elle intervient à trois niveaux : (a) la liaison de l'ARNm par l'intermédiaire de sa séquence «Shine-Dalgarno ». Cette liaison est dirigée par le facteur d'initiation IF3 [6] ; (b) l'attachement de l'anticodon de l'aminoacyl-ARNt initiateur 
(fMet-ARNt ${ }^{\mathrm{F}}$ ) au codon d'initiation AUG de l'ARNm, catalysé par le facteur IF2; et (c) la fixation de l'anticodon d'un deuxième aminoacyl-ARNt (AA-ARNt) au codon correspondant. Divers antibiotiques, dont le site de liaison est localisé sur la petite sous-unité, peuvent interférer avec une ou plusieurs fonctions de celle-ci : les aminoglycosides, les tétracyclines, l'édéine et la colicine B (Tableau II).

Les aminoglycosides. Les membres les plus connus de cette famille sont : (a) la néomycine et la paromomycine ; (b) les kanamycines, les gentamicines et la tobramycine; (c) la streptomycine, la kasugamycine, l'hygromycine et la spectinomycine. Les aminoglycosides produisent essentiellement trois types d'effet toxique sur la cellule microbienne [7]: (a) une altération de la membrane, qui se traduit par une modification de la perméabilité ; (b) une inhibition du métabolisme des acides nucléiques ( $\mathrm{ADN}$ et $\mathrm{ARN}$ ) ; et (c) un effet sur la synthèse des protéines (arrêt complet ou formation de protéines erronées). La streptomycine, remède antituberculeux bien

Tableau II

MODE D'ACTION DES PRINCIPAUX INHIBITEURS

DE LA TRADUCTION CHEZ LES PROCARYOTES

\begin{tabular}{|c|c|c|}
\hline Inhibiteur & Étape & Mode d'action \\
\hline Streptomycine & Initiation & Erreur de lecture \\
\hline Kasugamycine & Initiation & Déstabilise la liaison du fMet-ARNt \\
\hline $\begin{array}{l}\text { Colicine E3 } \\
\text { Édéine }\end{array}$ & $\begin{array}{l}\text { Initiation } \\
\text { Élongation }\end{array}$ & $\begin{array}{l}\text { Clive l'extrémité } 3 \text { ' de l'ARNr } 16 \mathrm{~S} \\
\text { Inhibe la liaison des substrats aux } \\
\text { sites } A \text { et } P\end{array}$ \\
\hline Tétracyclines & Élongation & $\begin{array}{l}\text { Inhibe la liaison de I'AAM-ARNt au } \\
\text { site } A\end{array}$ \\
\hline Puromycine & Élongation (DPT) & $\begin{array}{l}\text { Agit comme un analogue de I'AA- } \\
\text { ARNt }\end{array}$ \\
\hline Chloramphénicol & Élongation (DPT) & $\begin{array}{l}\text { Influence la cinétique de formation } \\
\text { du lien peptidique }\end{array}$ \\
\hline $\begin{array}{l}\text { Erythromycine et } \\
\text { Streptogramines B }\end{array}$ & Élongation (DPT) & $\begin{array}{l}\text { Influencent la formation du lien pep- } \\
\text { tidique via le site } P\end{array}$ \\
\hline Streptogramines $\mathrm{A}$ & Élongation (DPT) & $\begin{array}{l}\text { Interfèrent avec la liaison des subs- } \\
\text { trats aux } 2 \text { sites du DPT }\end{array}$ \\
\hline Acide fusidique & Élongation & $\begin{array}{l}\text { Bloque le facteur EF-G sur le ribo- } \\
\text { some. }\end{array}$ \\
\hline $\begin{array}{l}\text { Thiostreptone } \\
\text { Kirromycine }\end{array}$ & $\begin{array}{l}\text { Élongation } \\
\text { Élongation }\end{array}$ & $\begin{array}{l}\text { Empêche la liaison du facteur EF-G } \\
\text { Bloque le facteur EF-Tu sur le ribo- } \\
\text { some }\end{array}$ \\
\hline
\end{tabular}

DPT est l'abréviation de * domaine de la peptidyl-transférase • qui englobe le site de liaison de l'aminoacyl-ARNt, celui du peptidyl-ARNt ainsi que le centre catalytique.

$\mathrm{m} / \mathrm{s} n^{\circ} 1$ vol. 6, janvier 90

Tableau 1

SPÉCIFICITÉ RIBOSOMIQUE DES PRINCIPAUX INHIBITEURS DE LA TRADUCTION

\begin{tabular}{|c|c|c|}
\hline 305 & $40 S$ & $305 / 40 S$ \\
\hline $\begin{array}{l}\text { Streptomycine } \\
\text { Spectinomycine } \\
\text { Kasugamycine } \\
\text { Colicine E3 }\end{array}$ & $\begin{array}{l}\text { Émétine } \\
\text { Tubulosine } \\
\text { Tylophorine }\end{array}$ & $\begin{array}{l}\text { Pactamycine } \\
\text { Édéine }\end{array}$ \\
\hline $50 S$ & 605 & $50 S / 60 S$ \\
\hline $\begin{array}{l}\text { Chloramphénicol } \\
\text { Macrolides } \\
\text { Érythromycine } \\
\text { Carbomycine } \\
\text { Spiramycine } \\
\text { Tylosine }\end{array}$ & $\begin{array}{l}\text { Cycloheximide } \\
\text { Anisomycine } \\
\text { Abrine } \\
\text { Ricine } \\
\text { Sarcine alpha }\end{array}$ & $\begin{array}{l}\text { Puromycine } \\
\text { Sparsomycine } \\
\text { Acide fusidique } \\
\text { Amicétine } \\
\text { Blasticidine S } \\
\text { Bottromycine }\end{array}$ \\
\hline $\begin{array}{l}\text { Lincosamides } \\
\text { Lincomycine } \\
\text { Clindamycine }\end{array}$ & $30 S / 50 S$ & $\begin{array}{l}30 S / 50 S \\
40 S / 60 S\end{array}$ \\
\hline $\begin{array}{l}\text { Streptogramines } \\
\text { Pristinamycines I } \\
\text { Pristinamycines II } \\
\text { Vernamycines } \\
\text { Staphylomycines } \\
\text { Virginiamycines } \\
\text { Mikamycines }\end{array}$ & $\begin{array}{l}\text { Aminoglycosides } \\
\text { Gentamycine } \\
\text { Kanamycine } \\
\text { Néomycine }\end{array}$ & Tétracyclines \\
\hline Thiostreptone & Viomycine & \\
\hline
\end{tabular}

Les principaux inhibiteurs de la biosynthèse des protéines sont classés selon la nature de la cible. Un grand nombre montre une action très sélective, dans la mesure où ils agissent exclusivement sur les ribosomes des procaryotes ou eucaryotes; par contre, d'autres peuvent interférer avec les différents types de ribosome. D'après la référence [2].

connu, induit dans la cellule microbienne ces trois types d'effet. La formation d'un complexe $30 \mathrm{~S}$. streptomycine empêche la fixation d'ARNm et de fMet-ARNt et bloque ainsi l'initiation de la traduction. L'induction d'erreurs de lecture a été attribuée à la fixation de l'antibiotique sur la protéine $\mathrm{S} 12$ de la sousunité $30 \mathrm{~S}$, laquelle contrôle la fidélité d'appariement codon-anticodon. L'acheminement vers la membrane des faux peptides ainsi produits augmenterait sa perméabilité et entraînerait un blocage de tous les ribosomes, via un processus autocatalytique [7]. $\mathrm{Ni}$ la kasugamycine, un inhibiteur spécifique de l'initiation, ni la 


\section{RÉFÉRENCES}

1. Vazquez D. Inhibitors of proteins synthesis. Molecular biology biochemistry and biophysics, vol. 30. Berlin, Heidelberg, New York : Springer Verlag, 1979 : 312

2. Gale EF, Cundliffe E, Reynolds PE, Richmond MH, Waring MJ. The molecular basis of antibiotic action, 2nd ed. London, New York, Sydney, Toronto : Wiley, 1981 : 646.

3. Pestka S. Insights into protein biosynthesis and ribosome function through inhibitors. Progr Nucleic Acid Res Mol Biol 1976; 17 : 217-45.

4. Di Giambattista M, Cocito C. Le ribosome bactérien : structure et fonctions. médecine/ sciences 1989 ; 5 : 662-9.

5. Moore PB. The ribosome returns. Nature 1988 ; 331 : 223-7.

6. Steitz JA, Jakes K. How ribosomes select initiator regions in mRNA : base pair formation between the 3' terminus of 16S rRNA and the mRNA during initiation of protein synthesis in E. coli. Proc Natl Acad Sci USA $1975 ; 72: 4734-8$.

7. Davis BD. Mechanism of bactericidal action of aminoglycosides. Microbiol Rev 1987 ; $51: 341-50$.

8. Davies J, Smith Di. Plasmid-determined resistance to antimicrobial agents. Ann Rev Microbiol 1978; 32 : 469-518.

9. Cannon M, Cabezon T, Bollen A. Mapping of neamine resistance: identification to two genetic loci, nea A and nea B. Molec. Gen. Genet. 1974; 130 : 321-324.

10. Bückel $\mathrm{P}$, Bucheberg $\mathrm{A}$, Böck A, Wittmann MG. Alteration of ribosomal protein L6 in mutants of E. coli resistant to gentamycin. Molec. Gen. Genet. 1977 ; 158 : 47-54.

11. Ozaki M, Mizushima S, Nomura M. Identification and functional characterization of the protein controlled by the streptomycinresistant locus in E. coli. Nature 1969; 222 : 333-339.

12. Bollen A, Davies J, Ozaki M, Mizushima S. Ribosomal protein conferring sensitivity to antibiotic spectinomycin in E. coli. Science $1969 ; 165: 85-86$

13. Moazed D, Noller HF. Interaction of antibiotics with functional sites in 165 ribosoma RNA. Nature 1987 ; 327 : 389-94.

* La résistance est plasmidique lorsqu'elle es due à l'expression d'un gène porté par un plasmide, élément génétique extrachromosomique à réplication autonome; elle est dite chromosomique lorsqu'elle est expliquée par l'action ou la mutation d'un gène localisé dans le chromosome bactérien. spectinomycine, un inhibiteur de la translocation, n'induisent l'accumulation de polypeptides erronés, alors que la néomycine cause des erreurs de lecture et bloque la translocation [2]. Lorsque des bactéries sensibles sont cultivées en présence de streptomycine, on peut observer l'apparition de mutants dépendants et de mutants résistants à l'antibiotique. La plupart des mutants résistants aux aminoglycosides contiennent des plasmides codant pour des enzymes de phosphorylation, d'acétylation ou d'adénylation [8]. Dans les cas de résistance chromosomique $^{*}$, des mutations au niveau des gènes codant pour les composants ribosomaux, ARNr $16 \mathrm{~S}$ et protéines $S$, ont été décrites. En effet, des modifications dans les protéines S5 (spectinomycine), S6 (kanamycine), S12 (streptomycine), Sl2 et Sl7 (néamine) et L6 (gentamycine) ont été mises en évidence. Moazed et Noller [13] ont analysé les effets protecteurs exercés par certains aminoglycosides sur les modifications chimiques de l'ARNr 16S. Les sites de fixation de la streptomycine (bases A913-915 de l'ARNr 16S), de la spec- tinomycine (Cl063), de la néomycine, paromomycine, gentamycine et kanamycine (Al408 et Gl494) ont ainsi été identifiés (Tableau III). Il est opportun de noter que les bases Al408 et G1494, également protégées par l'ARNm dans l'approche suivie par Noller, semblent faire partie du site A (site de décodage).

Ces conclusions se voient confirmées par les résultats du marquage d'affinité du ribosome à l'aide de la streptomycine [14] et de l'analyse des mutants de résistance aux aminoglycosides [15].

Les tétracyclines. Les tétracyclines, de large emploi thérapeutique, se fixent aux sous-unités $30 \mathrm{~S}$ et sont considérées comme des inhibiteurs du site A [1]. En fait, la liaison du complexe aminoacyl-ARNt.EFTu.GTP aux ribosomes programmés (70S.ARNm), est inhibée par les tétracyclines. Ces mêmes antibiotiques interfèrent aussi avec la terminaison, une étape se déroulant également au niveau du site A. Des expériences récentes ont identifié la base A892 de l'ARNr 16S comme faisant partie du site de liaison de ces antibiotiques [13]. Cela implique une

\begin{tabular}{|l|c|c|c|l|}
\hline \multicolumn{5}{|c|}{ SITES DE LIAISON DES ANTIBIOTIQUES ET FONCTIONS } \\
RIBOSOMIQUES ASSOCIEES
\end{tabular}

Les différentes mutations ainsi que les résultats de protection qui ne sont pas repris dans ce Tableau se trouvent sur la figure 2. 
proximité des régions 890 et 1492 au sein de la structure tridimensionnelle de l'ARNr 16S (Tableau III). Il est opportun, toutefois, de citer le cas d'une mutation dans la protéine S10, laquelle serait responsable de la résistance chromosomiale aux tétracyclines [2]

L'édéine. Cet antibiotique inhibe l'attachement du fMet-ARNt au complexe 30S.ARNm: l'initiation est ainsi bloquée. Comme les tétracyclines, l'édéine interfère avec l'élongation des chaînes peptidiques, mais au niveau du site $P$. Elle est de ce fait considérée comme un inhibiteur spécifique de la fonction de ce site [16]. Signalons enfin que les bases A794/C795 et G926 font partie du site de fixation de l'édéine (Tableau III) et donc du site $\mathrm{P}$ des ribosomes.

La colicine E3. Contrairement à la plupart des antibiotiques, qui sont de petites molécules agissant de façon stœchiométrique, les colicines sont des protéines de haut poids moléculaire dont l'action est catalytique. La colicine E3 doit être considérée comme une 'endoribonucléase spécifique, clivant l'ARNr $16 \mathrm{~S}$ à proximité de son extrémité 3'-terminale [17, 18] (figure 1). Rappelons que l'hypothèse d'un appariement entre l'ARNr $16 \mathrm{~S}$ et la séquence Shine-Dalgarno de l'ARNm a été vérifiée grâce à l'utilisation de la colicine E3 [6].

\section{Antibiotiques inhibiteurs de la grande sous-unité ribosomique}

Au cours de la synthèse protéique, la sous-unité $50 \mathrm{~S}$ se lie au complexe d'initiation (ARNm.30S.fMetARNt) : les facteurs d'initiation sont recyclés et la phase d'élongation débute. Celle-ci comporte trois étapes : (a) l'attachement de l'aminocyl-ARNt (AA-ARNt) au site A, dirigé par le facteur EF-Tu; (b) la formation du lien peptidique entre le fMet-ARNt au site $P$ et l'AA-ARNt au site A, catalysée par la peptidyltransférase ; et (c) la translocation du peptidyl-ARNt, du site $A$ au site $P$, grâce au facteur EF-G. La majorité des antibiotiques agissant à ce niveau (macrolides, lincosamides, $\mathrm{m} / \mathrm{s} n^{\circ} 1$ vol. 6 , janvier 90 synergimycines, ou streptogramines et chloramphénicol) inhibent la formation du lien peptidique (Tableau II). On pourrait les classer en inhibiteurs du site accepteur, du site donneur ou du centre catalytique de l'enzyme, mais il est opportun, à ce stade, de mentionner que certains antibiotiques interfèrent avec les deux sites à la fois. Ce comportement peut s'expliquer à l'aide d'un nouveau modèle de la peptidyl-transférase qui prévoit un échange alternatif des positions et fonctions des sites donneur et accepteur de l'enzyme [19].

La puromycine. Cet antibiotique est devenu un outil essentiel en biologie moléculaire. En tant qu'analogue structural du phénylalanyl-ARNt, il se lie au site $A$; la réaction de peptidisation qui s'ensuit aboutit à la formation de peptidyl-puromycine [20]. Le détachement des chaînes polypeptidiques incomplètes explique l'inhibition de la synthèse protéique se produisant in vivo en présence de l'antibiotique. Dès lors, la puromycine est considérée comme un réactif de choix de la réaction de transfert de peptidyles et de l'identification des sites A et P. En effet, un peptidyl-ARNt confiné au site A ne peut réagir avec la puromycine : cette réaction devient en revanche possible à la suite de l'addition de EF-G et du GTP, qui produisent la translocation de peptidyl-ARNt au site $P$. Dans le modèle appelé « réaction du fragment », un oligonucléotide porteur d'un acide aminé acylé (tel le CCA-fMet représentant l'extrémité 3' $\mathrm{OH}$ de l'ARNt initiateur) réagit avec la puromycine en présence de la sous-unité ribosomique $50 \mathrm{~S}$ et donne lieu à la formation de la fMet-puromycine [21]. Le chloramphénicol et certains antibiotiques du groupe MLS (voir glossaire, p. 54), qui bloquent la synthèse de peptidyl-puromycine et la réaction du fragment, sont considérés comme des inhibiteurs de la peptidyl-transférase [21] Les protéines L11, L23 et S14 ainsi qu'un segment de l'ARNr23S feraient partie du site d'attachement de la puromycine sur le ribosome $70 S$ [2].

Le chloramphénicol. Le chloramphénicol, qui fut le premier antibiotique à large spectre produit par synthèse et utilisé en thérapeutique, est considéré comme l'inhibiteur de référence de la peptidyl-transférase. Cet antibiotique inhibe, dans les systèmes acellulaires, la synthèse protéique dirigée par des messagers naturels ou synthétiques, et, dans la «réaction du fragment », la formation de la liaison peptidique [21]. Un certain nombre de protéines de la sous-unité $50 \mathrm{~S}$ (L2, L4, L15, L16, L18 et L27) ont été localisées dans le domaine présumé de la peptidyltransférase. Les protéines identifiées par marquage d'affinité à l'aide de divers analogues du chloramphénicol ont été : L2, L6, L16, L24 et L27 [2]. Des recherches récentes [22] montrent un effet protecteur exercé par le chloramphénicol sur les nucléotides A245l et G2505 de l'ARNr 23S. De nombreuses mutations, conférant la résistance au chloramphénicol (G2447, A2451, C2452, A2503 et U2504) ou aux antibiotiques du groupe MLS (figure 2) laissent penser que la boucle de la région V de l'ARN 23S (bases 24502610) fait partie intégrante du domaine de la peptidyl-transférase, dont le centre catalytique constituerait le site de fixation du chloramphénicol.

Les macrolides. Les macrolides, couramment utilisés en médecine, se composent de plusieurs sous groupes selon le nombre d'atomes de leur macrocycle lactonique : $12\left(\mathbf{M}_{12}\right.$, méthymycine), $14\left(M_{14}\right.$, érythromycine, oléandomycine) ou à 16 atomes $\left(\mathbf{M}_{16}\right.$, carbomycines, leucomycines, spiramycines, tylosines). Seuls les dérivés $M_{16}$ inhibent la synthèse protéique dans le système acellulaire de Nirenberg, la réaction à la puromycine et la réaction du fragment [21] Des travaux récents ont montré, toutefois, que l'érythromycine (dont le mécanisme d'action est semblable à celui des synergimycines B) produit in vitro une nette inhibition de la synthèse protéique dirigée par des copolymères et un détachement prématuré des chaînes peptidiques en formation (voir les synergimycines de type B) [23, 24]. Les macrolides sont donc des inhibiteurs du domaine de la peptidyl-transférase.

Un type commun de résistance aux macrolides, lincosamides et synergistines de type $B$ est très répandu au 


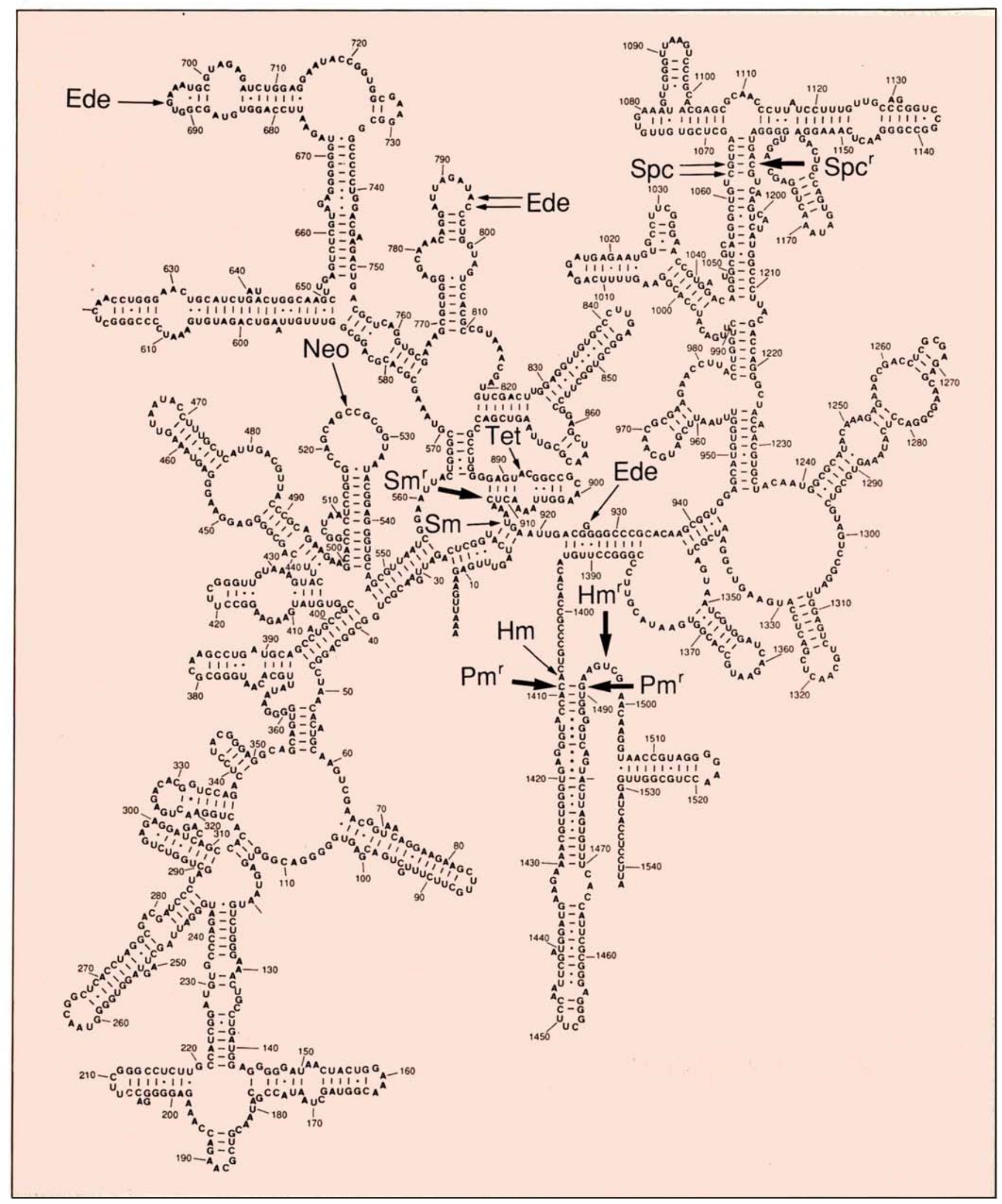

Figure 1. Modèle de la structure secondaire de I'ARN 165 de E. coli [44]. Les mutations conférant à divers organismes la résistance aux inhibiteurs de la petite sous-unité ribosomique sont signalées par une flèche grasse. Les bases, dont la réactivité vis-à-vis de certains agents chimiques est modifiée par la présence de divers antibiotiques sur le ribosome, sont signalées par une flèche fine (Tableau III). Ede =édéine; Spc = spectinomycine; Sm = streptomycine; Neo = néomycine ; 
sein des procaryotes (phénotype « MLS»). La résistance à ces trois familles, lorsque sélectionnée au laboratoire, peut être due à la modification des protéines ribosomales L4 ou L22 [25] ou de l'ARNr 23S (voir tableau III) [26-29].

Cette analyse génétique est confirmée par la protection des bases A2058/59 et G2505 de l'ARNr 23S, exercée par l'érythromycine, et celle des bases A2058/59/62 et G2505 du même ARNr, par la carbomycine [22] : ces bases appartiennent à la boucle de la région $\mathrm{V}$ de l'ARNr 23S, que l'on situe dans le domaine de la peptidyl-transférase). En revanche, la résistance naturelle (souches isolées en milieu hospitalier, organismes producteurs des MLS) est souvent associée à la diméthylation de l'A2058 de l'ARN 23S [30, 31]. Ce type de résistance, ses mécanismes de régulation et son évolution ont été très largement décrits [32, 33, 34].

Les lincosamides ont un mécanisme d'action semblable aux macrolides et partagent la même cible (compétition entre les MLS lors de la liaison au ribosome). Divers mutants de $E$. coli, résistants à la lincomycine ont été isolés. La résistance est liée à la modification des protéines ribosomales S7, L4 et L15 [35] ou, comme déjà mentionné plus haut dans le cas des macrolides, à des modifications dans l'ARN 23S.

Les synergimycines. Les antibiotiques de cette famille (mikamycines, pristinamycines, synergistines, streptogramines, vernamycines, virginiamycines) contiennent deux types de composés, $\mathrm{A}$ et $\mathrm{B}$, qui agissent en synergie. Séparément, $A$ et $B$ possèdent un pouvoir bactériostatique, alors que le mélange $\mathrm{A}+\mathrm{B}$ est jusqu'à cent fois plus actif que ses composants séparés et peut se révéler bactéricide [36]. Quelles sont les bases moléculaires du synergisme des composés $A$ et $B$ ? On sait que les ribosomes, incubés avec les dérivés $A$, fixent les composés B avec une constante d'affinité augmentée environ 10 fois [36]. Des expériences de cinétique rapide ont permis d'expliquer ce phénomène en postulant un changement conformationnel du ribosome induit par la liaison des dérivés du type A [41]. Les synergimycines du type A peuvent être $\mathrm{m} / \mathrm{s} n^{\circ} 1$ vol. 6, janvier 90

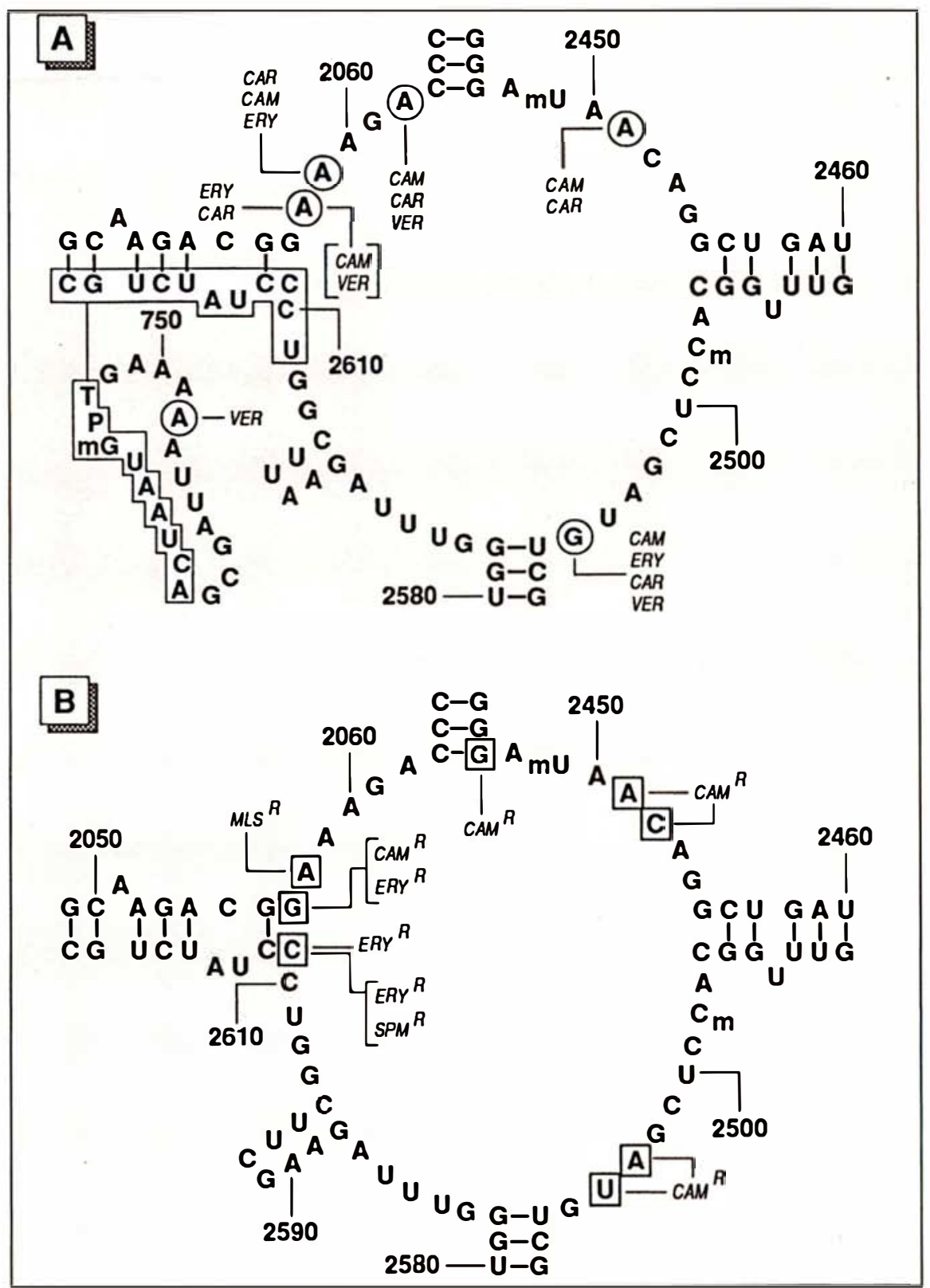

Figure 2. Portion (boucle V) de la structure secondaire de I'ARN 235 de E. coli [44]. Les bases, dont la réactivité, vis-à-vis de divers agents chimiques, est modifiée, sont répertoriées dans la partie $A$. Les mutations conférant la résistance à divers inhibiteurs du domaine de la peptidyl-transférase (chloramphénicol et MLS) sont reprises dans partie $B$ (Tableau III). une portion de la région II de I'ARN 23S (730 à 760) est également représentée en A. Les séquences encadrées semblent, en effet, se situer à proximité, au sein de la structure du ribosome, comme l'indiquent les résultats de divers pontages et la présence de la base A752, protégée par la vernamycine $B$. MLS = macrolides, lincosamides et streptogramines B; CAM $=$ chloramphénicol ; ERY = érythromycine ; CAR = carbomycine $;$ VER = vernamycine $B ; S P M=$ spiramycine. L'indice supérieur (r) indique la résistance à l'antibiotique.

considérées comme des inhibiteurs de la peptidyl-transférase, dont l'action se situe tant au site A qu'au site $P$ [37]. Tout en n'ayant aucune influence sur la liaison enzymatique de l'AA-ARNt au site A, elles induisent en revanche son décrochage.
Elles favorisent aussi l'éjection du peptidyl-ARNt du site $\mathbf{P}$ par le biais de la translocation. En l'absence d'inhibiteurs, les dérivés acylés d'ARNt se lient normalement de façon stable aux sites donneur et accepteur de la peptidyl-transférase 


\section{RÉFÉRENCES}

14. Gravel M, Melançon P, Brakier-Gingras L. Crosslinking of streptomycin to the $16 \mathrm{~S}$ ribosomal RNA of Escherichia coli. Biochemistry 1987 ; $26: 6227-32$.

15. Thompson J, Skeggs PA, Cundliffe E. Methylation of $16 \mathrm{~S}$ ribosomal RNA and resistance to the aminoglycoside antibiotics gentamicin and kanamycin determined by DNA from the gentamicin-producer Micromonospora purpurea. Mol Gen Genet 1985; 201 : 168-73.

16. Szer W, Kurylo-Borowska Z. Effect of edeine on aminoacyl-tARN binding to ribosomes and its relationship to ribosomal binding site. Biochem Biophys Acta 1970; 224 : 477-86.

17. Bowmann CM, Dahlberg JE, Ikemura T, Konisky J, Nomura M. Specific inactivation of $16 \mathrm{~S}$ rRNA induced by colE3 in vivo. Proc Natl Acad Sci USA 1971; 68 : 964-8.

18. Senior BW, Holland IB. Effect of colE3 upon the $30 \mathrm{~S}$ ribosomal subunit. Proc Natl Acad Sci USA 1971 ; 68 : 959-63.

19. Chinali G, Di Giambattista M, Cocito C. Ribosome protection by IRNA derivatives against inactivation by virginiamycin M. Evidence for 2 types of interaction of IRNA with the donor site of the peptidyltransferase. Biochemistry $1987 ; 26$ : 1592-7.

20. Traut RR, Monro RE. The puromycin reaction and its relation to protein synthesis. J Mol Biol 1964 ; 10 : 63-72.

21. Monro RE, Vazquez D. Ribosome-catalyzed peptidyl-transfer : effect of some inhibitors of protein synthesis. J Mol Biol 1967 ; 28 : 161-5.

22. Moazed D, Noller HF. Chloramphenicol, erythromycin, carbomycin and vernamycin $B$ protect overlapping sites in the peptidyl transferase region of $\varepsilon_{J} S$ ribosomal RNA. Biochimie 1987 ; 69 : 879-84.

23. Chinali G, Nyssen E, Di Giambattista M, Coccito $C$. Inhibition of polypeptide synthesis in cell-free systems by virginiamycin $\mathrm{S}$ and erythromycin. Evidence for a common mode of action of type B synergimycins and 14. membered macrolides. Biochim Biophys Acta $1989 ; 949: 71-8$.

24. Menninger JF, Otto D.P. Erythromycin, carbomycin and spiramycin inhibit protein synthesis by stimulating the dissociation of peptidyl-tRNA from ribosomes. Anti. Agents and Chemo. $1982 ; 21: 811-818$.

25. Wittman MG et al. Biochemical and genetic studies on two different types of erythromycin resistant mutants of $\mathrm{E}$. coli with altered ribosomal protein. Molec. Gen. Genet.

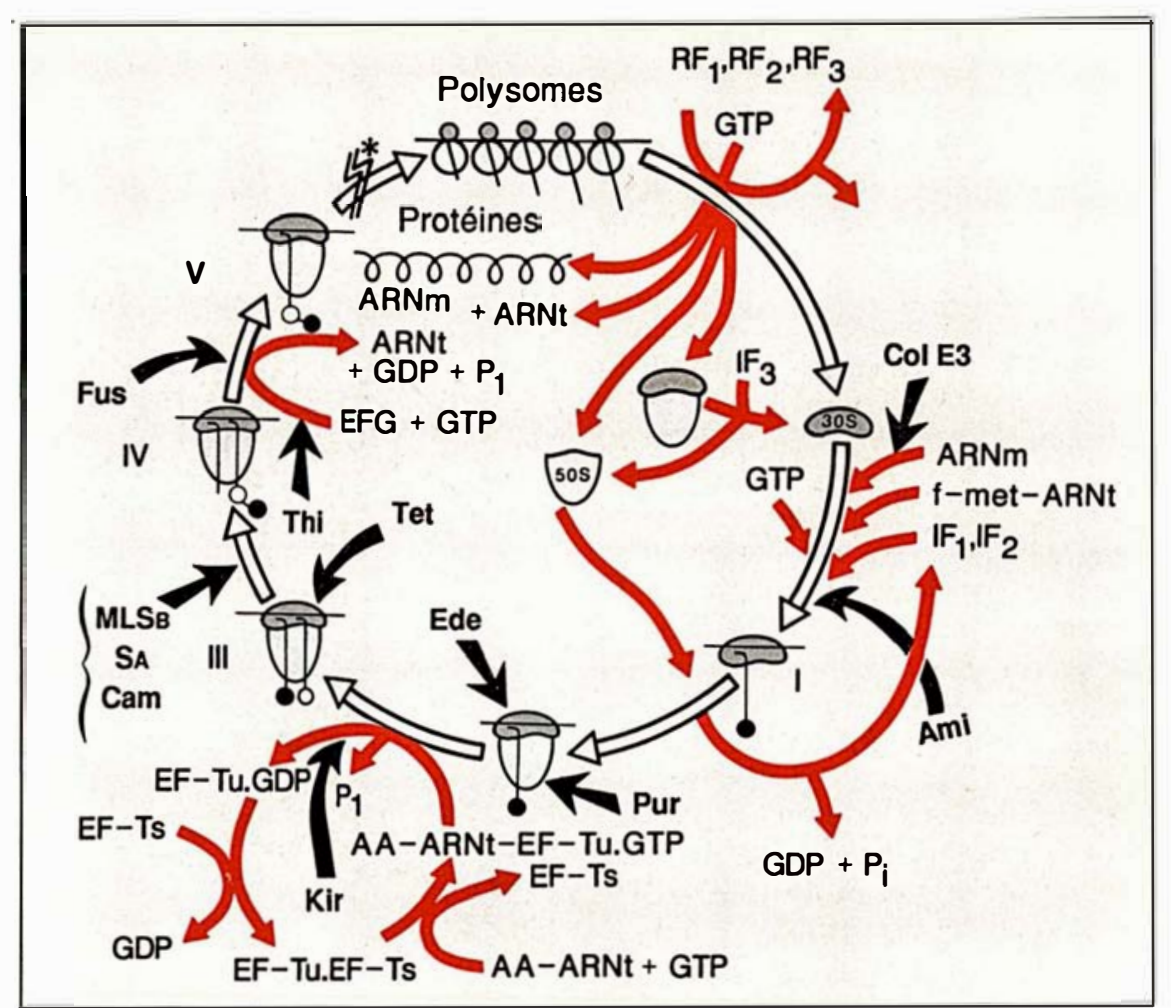

Figure 3. Le cycle ribosomal et les principaux inhibiteurs de la biosynthèse des protéines. L'action des principaux antibiotiques, interférant avec la biosynthèse des protéines, est schématisée par les flèches rouges. Col E3 = colicine E3 $;$ Ami = aminoglycosides $;$ Pur = puromycine $;$ Ede $=$ édéine $;$ Kir $=$ kirromycine; Tet $=$ tétracycline $; M L S_{B}=$ macrolides, lincosamides et streptogramines du type B; SA = streptogramines du type A ; Cam = chloramphénicol; Thi $=$ thiostreptone $;$ Fus $=$ acide fusidique.

alors que cette liaison est défavorisée en présence des synergimycines A [38, 39].

Selon des études récentes, les synergimycines de type B doivent aussi être considérées comme des inhibiteurs de la peptidyl-transférase [23]. De plus, ces antibiotiques stimulent le relâchement des chaînes peptidiques en formation au niveau d'acides aminés basiques [23]. Le site d'attachement des synergimycines $B$ a été localisé à la base de la protubérance centrale, l'emplacement présumé de la peptidyl-transférase [40]. Cette observation a été confirmée par les résultats d'expériences de protection exercée par la vernamycine $B$ sur les bases A752, A2062 et G2505 de l'ARNt $23 \mathrm{~S}$ vis-à-vis de l'action de divers agents chimiques [22]. Ces bases sont situées dans les boucles II et $\mathrm{V}$ de l'ARNr 23S. Des mutations conférant une résistance à ces antibiotiques ont été repérées dans la boucle de la région V [26] (figure 2).

\section{Antibiotiques inhibiteurs des facteurs d'élongation}

Certains antibiotiques interfèrent avec les étapes d'élongation en se liant soit aux facteurs d'élongation (la kirromycine, par exemple), soit à proximité des sites de EF-Tu et de EF-G sur la sous-unité ribosomique $50 \mathrm{~S}$ (acide fusidique et thiostreptone) (Tableau III).

La kirromycine, inhibiteur spécifique de l'EF-Tu, interfère avec la liaison de l'aminoacyl-ARNt (AAARNt) au site A, qui comporte plusieurs étapes: (a) liaison du complexe AA-ARNt. EF-Tu.GTP au site A ; (b) hydrolyse du GTP ; (c) détachement de EF-Tu.GDP ; et (d) fixation stable de l'AA-ARNt au site accepteur de la peptidyl-transférase. En présence de kirromycine (Kir), il y a formation d'un complexe AAARNt.GTP.EF-Tu.Kir qui se lie au site A et induit l'hydrolyse du GTP. 


\section{RÉFÉRENCES}

26. Cundliffe E. On the nature of antibiotic binding sites in ribosomes. Biochimie 1987 ; 69 : 863-9.

27. Skinner R, Cundliffe E, Schmidt FJ. Site of action of a ribosomal RNA methylase responsible for resistance to erythromycin and other antibiotics. J Biol Chem 1983 ; 258 : 12702-6.

28. Ettayebi M, Prasad SM, Morgan EA Chloramphenicol-erythromycin resistance mutations in a 23S rRNA gene of $E$. coli. $J$ Bacteriol 1985 ; 162 : 551-7.

29. Sigmund CD, Morgan EA. Erythromycin resistance due to a mutation in a ribosomal RNA operon of E. coli. Proc Natl Acad Sci USA 1971 ; 68 : 856-860.

30. Lai CJ, Weisblum B. Altered methylation of ribosomal RNA in an erythromycin-resistant strain of Staphylococcus aureus. Proc Natl Acad Sci 1971 ; 68 : 856-860.

31. Lai CJ, Weisblum B, Fannestock S R Nomura M. Alterations of 23S ribosomal RNA and erythromycin induced resistance to lincomycin and rifampicin in S. aureus. J Mol Biol 1973 ; 74 : 67-72.

32. Weisblum B. Inducible resistance to macrolides, lincosamides and streptogramin type $B$ antibiotics : the resistance phenotype, its biological diversity and structural elements that regulate expression. 1983 In Gene function in prokaryotes (ed. J. Deckwitter et al), p. 91 Cold Spring Harbor Laboratory, Cold Spring Harbor, New York.

33. Dubnau D. Translational alternation the regulation of bacterial resistance to the MLS antibiotics. CRC Crit. Rev. Biochem $1984 ; 16: 103-132$

34. Dubnau D, Monod $\mathrm{M}$. The regulation and evolution of MLS resistance p. 369-387. In K. P. Novick and S. B. Levy (ed) Evolution and environmental spread of antibiotic resis tance genes. Cold S. Harbor Labor. Cold Spring Harbor, New York.

35. Hummel M, Piepersberg W, Böck A Analysis of lincomycin resistance in E. coli. Mol Gen Genet 1979; 169 : 345-347.

36. Cocito C. Antibiotics of the virginiamycin family, inhibitors which contain synergistic components. Microbiol Reus 1979; 43 . 145-98.

37. Chinali G, Moureau P, Cocito C. The action of virginiamycin $\mathrm{M}$ on the acceptor, donor and catalytic sites of peptidyl-transferase. J. Biol Chem 1984; 259: 9563-9.

38. Parfait R, Cocito C. Lasting damage to bacterial ribosomes by reversibly-bound virginiamycin M. Proc Natl Acad Sci USA1980; 77 : 5492-6.

$\mathrm{m} / \mathrm{s} n^{\circ} 1$ vol. 6 , janvier 90
La séquence de réactions s'arrête à ce niveau ; le complexe étant bloqué sur le ribosome, l'AA-ARNt ne peut prendre part à la formation de la liaison peptidique [42].

L'acide fusidique, un des rares antibiotiques à structure stéroïdique, interfère avec la translocation qui dépend de l'EF-G. Dans des condiions physiologiques, la réaction de peptidisation aboutit à un complexe d'élongation porteur d'un peptidylARNt au site A et d'un ARNt déacylé au site P. La fixation de EF-G.GTP au site A entraîne l'hydrolyse du GTP et la translocation du peptidylARNt du site A au site $P$, avec éjection simultanée de l'ARNt déacylé. En se liant au ribosome, à proximite du site A, l'acide fusidique empêche la répétition de ces réactions nécessaires pour l'insertion de chaque acide aminé dans la chaîne peptidique en croissance [2].

La thiostreptone inhibe les fonctions des deux facteurs d'élongation EFTu et EF-G. Ce double effet s'explique par une impossibilité de liaison simultanée des facteurs EF-Tu et EF$\mathrm{G}$ au ribosome. Le recouvrement partiel de leurs sites de fixation explique qu'ils puissent subir des changements conformationnels conjoints à la suite de la liaison de la thiostreptone. Cet antibiotique a permis, notamment, de mettre en évidence un centre unique d'hydrolyse du GTP à la surface de la sous-unité 50S. La protéine Lll se localiserait à proximité du site de fixation de l'antibiotique. En effet, des sous-unités $50 \mathrm{~S}$ dépourvues de $\mathrm{L} 11$ ne recouvrent la capacité de lier l'antibiotique que si on leur ajoute cette protéine. Des recherches récentes montrent que la thiostreptone interagit avec une région de l'ARNr $23 S$ dont des mutations confèrent une résistance à cet antibiotique [43] L'organisme qui produit la thiostreptone (Streptomyces azureus) possède une enzyme capable de méthyler certains pentoses de l'ARNr 23S. Les particules ribosomiques correspondantes ne fixent plus l'antibiotique et deviennent de ce fait insensibles à son action [26].

\section{Conclusions}

Les inhibiteurs de la biosynthèse des protéines présentent, non seulement un intérêt thérapeutique évident, mais leur utilisation dans les systèmes acellulaires de synthèse protéique a contribué à l'élucidation des mécanismes de la traduction.

$\mathrm{Au}$ travers des diverses spécificités d'action (nature de la cible, étape de la traduction, influence de la fidélité ou de l'efficience de la traduction), on peut constater qu'il existe autant de familles d'inhibiteurs que de réactions clefs dans le processus de traduction.

Les antibiotiques, que l'on peut considérer comme autant d'inhibiteurs enzymatiques, se sont avérés d'excellents outils, afin d'étudier les différents centres actifs du ribosome. Dans ce contexte, l'analyse des modifications ribosomales, rencontrées chez divers mutants résistants, s'est révélée très précieuse. En effet, de nombreuses mutations, conférant la résistance aux inhibiteurs de la traduction, se localisent au niveau des ARNr, alors que le rôle de ces derniers au sein du ribosome avait longtemps été réduit à un rôle strictement structural, les protéines ribosomales assurant, seules, les fonctions enzymatiques.

De même, les résultats de protection (vis-à-vis de divers agents chimiques) de certaines bases nucléotidiques des ARNr, en présence des antibiotiques, confirment les résultats génétiques et suggèrent fortement que l'ARN ribosomal serait la cible essentielle de ces inhibiteurs. Rappelons enfin que les nucléotides, désignés par les approches génétiques et biochimiques, se localisent dans les régions des ARNr fortement conservées au cours de l'évolution. La découverte que certaines molécules d'ARN peuvent être dotées d'activités enzymatique (autoreplication) et les résultats présentés dans cette synthèse suggèrent que l'ARNr jouerait un rôle fondamental dans la traduc. tion [26]

\section{TIRÉS A PART}

C. Cocito. 
39. Cocito C, Chinali G. Molecular mechanisms of virginiamycin-like antibiotics on bacterial cell-free systems for protein synthesis. J Antimier Chemother $1985 ; 16$ supp A : 35-52.

40. Di Giambattista M, Thielen A, Maassen J, Möller W, Cocito C. Localisation of virginiamycin $\mathrm{S}$ binding site on bacterial ribosome by fluorescence energy transfer. Biochemistry $1986 ; 25: 35440-7$.

41. Di Giambattista M, Nyssen E, Engelborghs $\mathrm{Y}$, Cocito $\mathrm{C}$. Kinetics of binding of macrolides, lincosamides and synergimycins to ribosomes. J Biol Chem 1987; 262 : 8591-

42. Parneggiani A, Swart GWM. Mechanism of action of kirromycin-like antibiotics. Ann Rev Microbiol 1985; 39 : 557-77.

43. Thompson J, Cundliffe E, Stark M Binding of thiostrepton to a complex of $23 \mathrm{~S}$. rRNA with ribosomal protein Lll. Eur $J$ Biochem 1979; 98 : 261-5.

44. Guttel RR, Weiser B, Woese CR, Noller HF. Progr Nucleic Acid Res Mol Biol 1985 $32: 155-216$.

\section{* GLOSSAIRE}

$S$ : protéine ribosomique de la petite sous-unité.

L: protéine ribosomique de la grande sous-unité.

MLS : macrolides, lincosamides et streptogramines ou synergimycines du type $B$.

Boucle V:portion de la structure secondaire de l'ARN $23 S$.

EF-Tu: facteur d'élongation Tu. EF-G : facteur d'élongation $G$.

Site $A$ : site accepteur du ribosome.

Site $\boldsymbol{P}$ : site donneur du ribosome.

Réaction du fragment: modèle expérimental de la réaction de formation de la liaison peptidique.

Domaine de la peptidyl-transférase: domaine situé sur la grande sous-unité ribosomique et englobant le site $A$, site $P$ et le centre catalytique de l'enzyme.

$\boldsymbol{M}_{12}, \boldsymbol{M}_{10} \boldsymbol{M}_{16}$ : macrolides dont le cycle lactonique est constitué de 12,14 ou 16 chainons.

GTP : guanosine triphosphate.

\section{Summary}

Antibiotics inhibiting protein synthesis

Recent works on : (1) the mechanisms of action of protein synthesis inhibitors, (2) the bases of microbial resistance to these drugs, and (3) the use of antibiotics as tools for probing structure and functions of ribosomes are reviewed. Three groups of inhibitors of the large (50S), the small (30S) ribosomal subunits, and the elongation factors (EF-Tu, EF-G) were considered. The $30 \mathrm{~S}$ subunit controls initiation (by binding mRNA) but also elongation (fidelity of codon-anticodon pairing); hence, the block of initiation and the misstranslation occurring in the presence of aminoglycosides. In addition, the occurrence of the $\mathrm{A}$ and $\mathrm{P}$ sites at the interface between the two subunits explains the interference of tetracyclines and edeine (both binding to 30S) with the functions of the former and latter site, respectively. The large subunit controls elongation: binding of AA-tRNA to A site, peptide bond formation between peptidyl-tRNA at $P$ site and AA-tRNA at A site, and translocation. Most 50S inhibitors (chloramphenicol, macrolides, lincosamides and synergimycins) inhibit the peptidization reaction, by binding to the peptidyltransferase domain (at the base of the central protuberance). Puromycin, as an AA-tRNA analog, binds to the A site : the ensuing peptidyltransfer reaction yields peptidylpuromycim. A model of this process (fragment reaction), used to probe peptidyltransferase function, is inhibited by some antibiotics listed above. Antibiotics may act either on elongation factors (formation of a kirromycin.EFTu complex), and still others by binding to the ribosomal site for EF-G attachment (thiostrepton and fusidic acid, translocational inhibitors). Chromosomal resistance to antibiotics, previously attributed to alterations of rProteins, is frequently referred to mutations of the genes for $16 \mathrm{~S}$ and $23 \mathrm{~S}$ rRNAs. 